\title{
Introduction to the Vol. 44, No. 2, 2017
}

\author{
Maomi Ueno
}

Published online: 14 September 2017

(C) The Behaviormetric Society 2017

Welcome to the Vol. 44, No. 2, 2017 of Behaviormetrika.

We renewed and expanded the journal subject areas and the editorial board organization in the Vol. 44, No. 1, 2017 (Ueno 2017). In the Vol. 44, No. 1, two special features were included: "Recent developments in causal discovery and inference" (Chalupka et al. 2017; Ma and Statnikov 2017; Henry and Gates 2017) which was edited by Shohei Shimizu, and "Probabilistic graphical models and its applications to biomedical informatics" (He et al. 2017; Liu et al. 2017; Taguri and Izumi 2017; Yamamoto et al. 2017) which was edited by Joe Suzuki and Brandon Malone. Both issues specifically addressed state-of-the-art methods of statistics, machine learning, and data science. The Vol. 44, No. 1 also includes one note paper (Kim et al. 2017), one review paper (Akama and Murphy 2017), and three original papers (Ogasawara 2017a, b, Suzuki 2017). After we renewed the journal, the flow of high-quality submissions to the journal have been increasing, but the acceptance rate remains relatively low (35\% in the Vol. 44, No. 2).

In this issue Vol. 44, No. 2, 2017, we have the following thirteen papers.

The original paper "Use of cognitive diagnostic model to study differential item functioning in accommodations" by Svetina et al. (2017) addresses differential item functioning (DIF), which is a statistical characteristic of an item that shows the extent to which the item might be measuring different abilities for members of separate subgroups, using a cognitive diagnostic model. This study explored potential sources of DIF among accommodated and non-accommodated groups by examining skills and cognitive processes hypothesized to underlie student performance on the National Assessment for Educational Progress (NAEP).

The original paper "Effectiveness of rank correlations in curvilinear relationships" by Tarsitano and Amerise (2017) analyzes the behavior of a few well-known

Maomi Ueno

ueno@ai.is.uec.ac.jp

1 University of Electro-Communications, Tokyo, Japan 
rank correlation coefficients, which is known to provide an efficient similarity measure when a non-linear connection exists, by focusing on some frequently encountered non-linear patterns. They conclude that a reasonably robust answer to the special needs arising from non-linear relationships could be given by a variant of the Fisher-Yates coefficient, which has a more marked tendency to reject the hypothesis of independence between pairs of rankings connected by several forms of non-linear interaction.

The original paper "Approximating score distributions using mixed-multivariate beta distribution" by Okubo and Mayekawa (2017) proposes a new smoothing empirical test score distribution. The problem is that the score distributions sometimes form a 'toothed' shape, meaning that the probability distribution does not always fit well. To solve the problem, they propose the maximum likelihood model for approximating a discrete distribution. Their approach is based on the mixed-multivariate beta distribution, which can cover asymmetric score distributions with known upper and lower bounds. Further, a simulation study is conducted to compare the results from the application of the mixed-multivariate beta distribution, mixed-multivariate normal distribution, and two polynomial log-linear models. Results show the effectiveness of the proposed method.

The original paper "An international research comparative study of the degree of cooperation between disciplines within mathematics and mathematical sciences: proposal and application of new indices for identifying the specialized field of researchers" by Mizukami et al. (2017) proposes new analysis methods of collaborative academic research activities and relationships in mathematics/mathematical science and 21 other fields using academic paper database. They focused on the "academic papers co-authored with researchers in other fields" and derived new methods for measuring the degree of research cooperation among disciplines. The methods consist of two steps. The first step involves measuring and deriving new indices for identifying the specialized field of each researcher and the degree of concentration of each researcher's work in his or her field of specialization. The second step, based on the indices, utilizes the Lorenz curve and Gini coefficient to identify the characteristics of each field.

The original paper "Identified and unidentified cases of the fixed-effects 3- and 4-parameter models in item response theory" by Ogasawara (2017c) shows unidentified cases of the fixed-effect 3- and 4-parameter models in item response theory although it has been believed to generally have the identification. The same phenomena have been reported by Maris (2008) and van der Linden and Barrett (2016). However, this paper demonstrates that identification is not to be guaranteed even when one of the other parameters is fixed. The results also sometimes have identified case when one of the other parameters is fixed. It is interesting because they are counter examples to van der Linden and Barrett (2016)'s unidentified results.

The original paper "Diverse reports recommendation system based on latent Dirichlet allocation" by Uto et al. (2017) proposes an educational intelligent system supporting learners in improving their report-writing skills by recommending reports from previous learners, based on social constructivism (Vygotsky 1978). The proposed system recommends reports that share similar subjects but which have 
different structures, expressions, and originality based on the distributions of words and subjects within the reports, as estimated using latent Dirichlet allocation (LDA). An important assumption of this study is that reports with different word distributions tend to include different structures, expressions, and originality when they share similar subjects. Based on that assumption, the proposed system selects and recommends reports that have dissimilar word distributions but which share similar subject distributions with a learner's report.

The Note paper "Logistic regression with misclassification in binary outcome variables: a method and software" by Liu and Zhang (2017) introduces a model with false-positive and false-negative misclassification parameters to deal with the influence of the misclassification. The proposed model can not only estimate the underlying association between the dependent and independent variables, but it also provides information on the extent of the misclassification. To estimate the model, a maximum likelihood estimation method based on a Fisher scoring algorithm is utilized. They conducted simulation experiments and demonstrates the effectiveness of the new model. An R package is developed to aid the application of the model.

The Note paper "How previous choice affects decision attribute weights: a field survey" by Hatori et al. (2017) examines the effects of people's prior choice making on their weights of decision attributes. According to theories on post-decision processes, preferences are likely to be reconstructed in line with a preceding choice. The present study hypothesized that preferences are constructed so that superior (inferior) attributes of a chosen alternative are weighted higher (lower) than before. This hypothesis was tested in a field survey of university students in Japan. The results support the hypothesis that the weights of the superior (inferior) attributes of the chosen alternative increased (decreased) as time passed since the decision was made.

Furthermore, the two papers are included in the second part of a special feature "Probabilistic graphical models and its applications to biomedical informatics," which is edited by Joe Suzuki and Brandon Malone. In Vol. 44, No. 1, 2017, we have already published four invited papers in part 1 as described before. Part 2 follows these papers, with the following two contributed papers.

The first original paper of the special feature is "Error asymmetry in causal and anticausal regression" by Blöbaum et al. (2017). This article theoretically analyzes the relationship between the intrinsic causality of two variables and the expected error. Specifically, it derives the theorem that the expected error of the true data generating function as prediction model is generally smaller when the effect is predicted from its cause and, on the contrary, greater when the cause is predicted from its effect. The theorem implies an asymmetry in the error depending on the prediction direction.

The second note paper of the special feature is "A note on large-scale logistic prediction: using an approximate graphical model to deal with collinearity and missing data" by Marsman et al. (2017). This paper proposes a novel approach to make accurate predictions of logistic regression models even when predictor variables are highly correlated and only partly observed. First they model the joint distribution of the outcome variable and the predictor variables using the Ising network model. Second, to render the application of Ising networks feasible, they 
use a latent variable representation to apply a low-rank approximation to the network's connectivity matrix. Finally, they approximate it to multivariate item response theory (MIRT) model to handle missing observation.

The second special feature of this issue is "Psychological science of survey questionnaires" which was guest edited by Kazuhisa Takemura and Michael Smithson. This special feature focuses on the psychological science of survey questionnaires to highlight important theoretical and empirical insights.

This special feature includes the following papers.

"Question word-order influences on covariate effects: predicting zero-sum beliefs" by Smithson et al. (2017).

"Coping with career development: a field study measuring the influence of work communication and employee personality" by Selart and Johansen (2017).

"How does response bias emerges in lengthy sequential preference judgments?" by Morii et al. (2017).

"Respondents with low motivation tend to choose middle category: survey questions on happiness in Japan" by Masuda et al. (2017).

We strongly recommend to read the preface of this special feature because Takemura and Smithson introduce each paper in their preface.

\section{References}

Akama H, Murphy B (2017) Emerging methods for conceptual modelling in neuroimaging. Behaviormetrika 44(1):117-133

Blöbaum P, Washio T, Shimizu S (2017) Error asymmetry in causal and anticausal regression. Behaviormetrika. doi:10.1007/s41237-017-0022-z

Chalupka K, Eberhardt F, Perona P (2017) Causal feature learning: an overview. Behaviormetrika 44(1):137-164

Hatori T, Fujii S, Takemura K (2017) How previous choice affects decision attribute weights: a field survey. Behaviormetrika. doi:10.1007/s41237-017-0028-6

He Y, Jia J, Geng Z (2017) Structural learning of causal networks. Behaviormetrika 44(1):287-305

Henry T, Gates K (2017) Causal search procedures for fMRI: review and suggestions. Behaviormetrika 44(1):193-225

Kim S, Cardwell R, Hwang H (2017) Using R Package gesca for generalized structured component analysis. Behaviormetrika 44(1):3-23

Liu H, Zhang Z (2017) Logistic regression with misclassification in binary outcome variables: a method and software. Behaviormetrika. doi:10.1007/s41237-017-0031-y

Liu S, Fukumizu K, Suzuki T (2017) Learning sparse structural changes in high-dimensional Markov networks. Behaviormetrika 44(1):265-286

Ma S, Statnikov A (2017) Methods for computational causal discovery in biomedicine. Behaviormetrika 44(1):165-191

Maris G (2008) A note on "Constant latent odds-ratios models and the Mantel-Haenszel null hypothesis". Psychometrika 73:153-157

Marsman M, Waldorp L, Maris G (2017) A note on large-scale logistic prediction: using an approximate graphical model to deal with collinearity and missing data. Behaviormetrika. doi:10.1007/s41237017-0024-X

Masuda S, Sakagami T, Kawabata H, Kijima N, Hoshino T (2017) Respondents with low motivation tend to choose middle category: Survey questions on happiness in Japan. Behaviormetrika. doi:10.1007/ s41237-017-0026-8

Mizukami Y, Mizutani Y, Honda K, Suzuki S, Nakano J (2017) An international research comparative study of the degree of cooperation between disciplines within mathematics and mathematical 
sciences: proposal and application of new indices for identifying the specialized field of researchers. Behaviormetrika. doi:10.1007/s41237-017-0023-y

Morii M, Sakagami T, Masuda S, Okubo S, Tamari Y (2017) How does response bias emerges in lengthy sequential preference judgments? Behaviormetrika. doi:10.1007/s41237-017-0036-6

Ogasawara H (2017a) Distribution-free properties of some asymptotic cumulants for the Mallows $C_{p}$ and its modifications in usual and ridge regression. Behaviormetrika 44(1):25-56

Ogasawara H (2017b) A family of the adjusted estimators maximizing the asymptotic predictive expected log-likelihood. Behaviormetrika 44(1):57-95

Ogasawara H (2017c) Identified and unidentified cases of the fixed-effects 3- and 4-parameter models in item response theory. Behaviormetrika. doi:10.1007/s41237-017-0032-x

Okubo T, Mayekawa S (2017) Approximating score distributions using mixed-multivariate beta distribution. Behaviormetrika. doi:10.1007/s41237-017-0019-7

Selart M, Johansen ST (2017) Coping with career development: A field study measuring the influence of work communication and employee personality. Behaviormetrika. doi:10.1007/s41237-017-0035-7

Smithson M, Shou Y, Yu A (2017) Question word-order influences on covariate effects: predicting zerosum beliefs. Behaviormetrika. doi:10.1007/s41237-017-0030-Z

Suzuki J (2017) A theoretical analysis of the BDeu scores in Bayesian network structure learning. Behaviormetrika 44(1):97-116

Svetina D, Dai S, Wang X (2017) Use of cognitive diagnostic model to study differential item functioning in accommodations. Behaviormetrika. doi:10.1007/s41237-017-0021-0

Taguri M, Izumi S (2017) A global goodness-of-fit test for linear structural mean models. Behaviormetrika 44(1):253-262

Tarsitano A, Amerise IL (2017) Effectiveness of rank correlations in curvilinear relationships. Behaviormetrika. doi:10.1007/s41237-017-0020-1

Ueno M (2017) As the oldest journal of data science. Behaviormetrika 44(1):1-2

Uto M, Louvigné S, Kato Y, Ishii T, Miyazawa Y (2017) Diverse reports recommendation system based on latent Dirichlet allocation. Behaviormetrika. doi:10.1007/s41237-017-0027-7

van der Linden WJ, Barrett MD (2016) Linking item response model parameters. Psychometrika 81:650-673

Vygotsky LS (1978) Mind in society: the development of higher psychological processes. Harvard University Press, Cambridge

Yamamoto M, Hirose K, Nagata H (2017) Graphical tool of sparse factor analysis. Behaviormetrika 44(1):229-250 